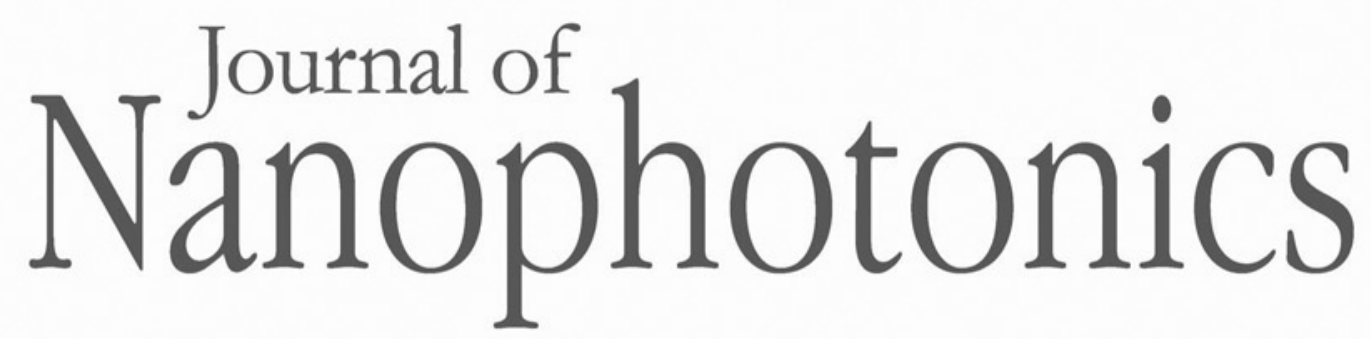

Nanophotonics.SPIEDigitalLibrary.org

\title{
Directional thermal emission from a leaky-wave frequency-selective surface
}

Edward C. Kinzel

James C. Ginn

Louis A. Florence

Brian A. Lail

Glenn D. Boreman

\section{SPIE.}




\title{
Directional thermal emission from a leaky-wave frequency-selective surface
}

\author{
Edward C. Kinzel, ${ }^{a} *$ James C. Ginn, ${ }^{b}$ Louis A. Florence, ${ }^{c}$ Brian A. Lail, ${ }^{d}$ \\ and Glenn D. Boreman ${ }^{\mathrm{e}}$ \\ ${ }^{a}$ Missouri University of Science and Technology, Department of Mechanical and Aerospace \\ Engineering, 400 West 13th Street, Rolla, Missouri 65409, United States \\ ${ }^{b}$ Plasmonics Inc., 12605 Challenger Parkway \#150, Orlando, Florida 32826, United States \\ ${ }^{\mathrm{c}}$ United States Military Academy, Physics and Nuclear Engineering Department, Building 735 \\ Science Center, West Point, New York 10996, United States \\ ${ }^{\mathrm{d}}$ Florida Institute of Technology, Electrical and Computer Engineering, 150 West University \\ Boulevard, Melbourne, Florida 32901, United States \\ ${ }^{\mathrm{e}}$ University of North Carolina at Charlotte, Physics and Optical Sciences, 9201 University City \\ Boulevard, Charlotte, North Carolina 28223, United States
}

\begin{abstract}
We design, fabricate, and characterize a frequency-selective surface (FSS) with directional thermal emission and absorption for long-wave infrared wavelengths. The FSS consists of an array of patch antennas connected by microstrips, the ensemble of which supports leakywave-type modes with forward and backward propagating branches. The branches are designed to intersect at $9.8 \mu \mathrm{m}$ and have a broadside beam with 20-deg full width at half maximum at this wavelength. The absorption along these branches is near unity. Measurement of the hemispherical directional reflectometer shows good agreement with simulation. The ability to control the spectral and directional emittance/absorptance profiles of surfaces has significant applications for radiation heat transfer and sensing. () The Authors. Published by SPIE under a Creative Commons Attribution 3.0 Unported License. Distribution or reproduction of this work in whole or in part requires full attribution of the original publication, including its DOI. [DOI: 10.1117/1.JNP.9.093040]
\end{abstract}

Keywords: frequency-selective surface; metasurface; thermal emission; infrared antenna; hemispherical directional reflectometer.

Paper 15071P received Jul. 29, 2015; accepted for publication Oct. 29, 2015; published online Dec. 14, 2015.

\section{Introduction}

A blackbody radiates the maximum amount of thermal energy into the far-field. The spectral distribution of this radiation is given by Planck's law and has a diffuse Lambertian profile without polarization selectivity. While real materials deviate from the blackbody response on the basis of their electromagnetic response (principally, permittivity at optical and infrared wavelengths) and surface roughness, the emitted radiation remains largely isotropic and with generally only gradual variations in the spectral distribution. ${ }^{1}$ These characteristics of natural surfaces result from the fact that the thermal radiation is incoherent. ${ }^{1-4}$

Controlling the angular distribution of thermal radiation has potential applications related to thermophotovoltaics, solar thermal energy collection, and thermal management. Controlling the angular distribution of thermal radiation requires a degree of coherence. This has been accomplished experimentally using polar materials ${ }^{2}$ and metallic gratings ${ }^{3}$ to produce very narrow angular distributions [less than 1-deg full width at half maximum (FWHM)]. This corresponds to long-coherence lengths of greater than $100 \mu \mathrm{m} .{ }^{3}$ These designs exhibit a leaky-wave/diffractive response with the angle of emission dependent on the wavelength. However, Cruz-Cabrera et al. $^{4}$ demonstrated directional emissivity near grazing angles of incidence over controlled wavelength intervals using subwavelength periodic two-dimensional array of resonators.

*Address all correspondence to: Edward C. Kinzel, E-mail: kinzele@mst.edu 
Frequency-selective surfaces (FSSs) consist of subwavelength metal elements. Control of the geometry and material selection allows the scattering parameters of the surface to be engineered. ${ }^{5}$ This makes FSS a type of metamaterial (a metasurface) with electromagnetic properties typically not found in nature. FSS has been used to control the spectral, ${ }^{6-8}$ phase, ${ }^{9-11}$ and polarization $^{12}$ responses of incident radiation. This is often achieved via scaling established radio frequency designs to infrared wavelengths ${ }^{13}$ which has significance at infrared wavelengths because it controls the emissivity of the surface, and hence heat transfer via radiation. For example, Liu et al. ${ }^{14}$ engineered the spectral emissivity of surface cross elements. In these cases, the angular distribution of the emitted radiation is still close to Lambertian.

Phased-array antennas are another RF concept that has been scaled to the IR. ${ }^{15-18}$ In these cases, two or more antenna elements are connected to a load using transmission lines. These devices have been used to generate a prescribed angular sensitivity for detectors, both thermopiles and metal-oxide-metal diodes. Significantly better response was reported from the diodes because of the distributed electromagnetic absorptance of the antennas and feed structure. ${ }^{18}$

In this paper, we design, fabricate, and test an FSS based on a patch antenna/microstripphased array to create a surface with angularly dependent thermal emission/absorption. The structure supports guided leaky-wave-type modes with forward and backward propagating branches coupled to transverse magnetic (TM)-polarized radiation. The choice of microstrip transmission lines leads to significantly better propagation lengths, and hence increased angular selectivity compared with previous coplanar strip designs. Because of metal losses at IR wavelengths, there is a distributed load throughout the FSS which differs from conventional RF design. However, after optimization, we are able to demonstrate a structure with high absorptance. These experimental results show reasonable spectral/directional agreement with the simulation. This work differs from previous reports because the thermal energy couples to the resonant patches and propagates along guided modes as opposed to via surface waves. In other words, it combines the functions of a leaky-wave antenna and a series array. The ability to achieve an angular response including near-unity absorption over a compact area may be useful for future sensors and emitters. This paper presents the design and fabrication procedures along with experimental results for the device.

\section{Device Geometry and Fabrication}

Figure 1 shows the geometry of the FSS. It was a designed and optimized structure for broadside (normal incidence, $\theta=0 \mathrm{deg}$ ) directional emission at $\lambda_{0}=9.72 \mu \mathrm{m}$. Conceptually, it consists of a series of patch antennas $(a \times b=2.157 \times 2.674 \mu \mathrm{m})$ connected by microstrip transmission lines $(w=0.730 \mu \mathrm{m})$. The pattern is separated from a gold ground plane by a $0.650-\mu \mathrm{m}$ standoff of zinc sulfide $(\mathrm{ZnS})$. Both the antennas and the ground plane are gold and are 75and 150-nm thick, respectively. The structure is periodic in both the $x$ - and $y$-directions $\left(p_{x} \times p_{y}=4.036 \times 4.3625 \mu \mathrm{m}\right)$.

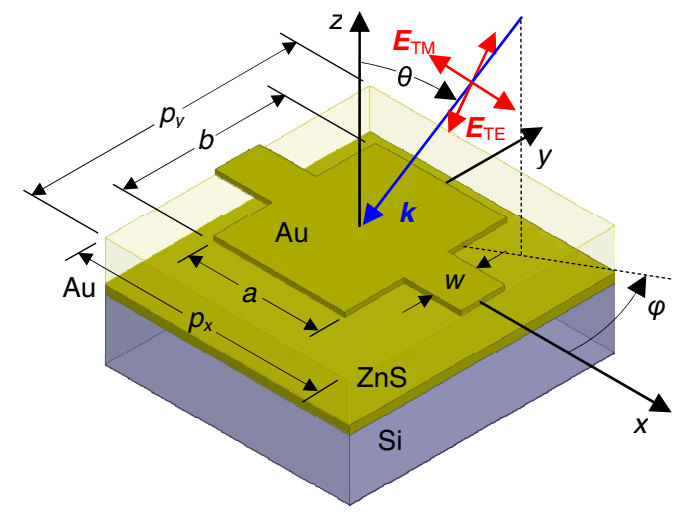

Fig. 1 Frequency-selective surface (FSS) unit cell and dimensions. 


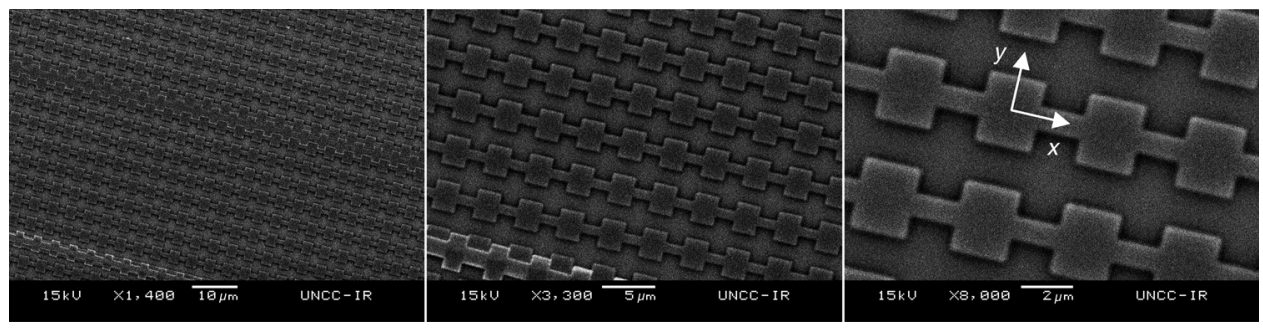

Fig. 2 Scanning electron microscope images of FSS.

ANSYS HFSS, a commercial finite-element method full-wave electromagnetic solver, was used to analyze and optimize the structure. The objective was to produce unity absorptance at $\lambda_{0}=9.72 \mu \mathrm{m}$ (peak emission at room temperature from Wien's displacement law: $\left.\lambda_{\max }=2898 \mu \mathrm{m} \cdot \mathrm{K} / T\right)$. Floquet port excitation of the unit cell shown in Fig. 1 was used to simulate the reflectance as a function of angle of incidence and polarization. Ellipsometery (J.A. Woollam IR VASE) was used to experimentally measure frequency-dependent material properties. These properties were used in the electromagnetic model to increase its fidelity. ${ }^{19}$ In optimizing the design, we specifically chose dimensions to minimize diffraction and surface plasmon polariton modes at the design wavelength. These modes occur when radiation is diffracted along the surface. The $m$ 'th mode will be cutoff when

$$
p<\frac{m \lambda}{1+\sin \theta_{i}},
$$

where $p$ is the periodicity, $\theta_{i}$ is the angle of incidence, and $m$ is the order. At long-wave infrared wavelengths, the surface plasmon has nearly the same wavelength as a free-space propagating wave and the energy is not tightly bound to the surface leading to long propagation lengths. All diffractive modes will be cutoff at $p<4.86 \mu \mathrm{m}$ for $\lambda=9.72 \mu \mathrm{m}$.

Figure 2 shows the scanning electron microscope images of the fabricated surface. E-beam evaporation was used to deposit the gold ground plane on a silicon substrate. The $\mathrm{ZnS}$ standoff was thermally evaporated on top of the ground plane. E-beam lithography lift-off was used to define the structure. The antennas were also deposited with e-beam evaporation and have a 5$\mathrm{nm}$-thick $\mathrm{Cr}$ adhesion layer (not included in simulation). We patterned a $2 \times 2 \mathrm{~cm}^{2}$ structure using lift-off and e-beam lithography. The relatively large area was required to measure the far-field using a hemispherical directional reflectometer (HDR). The vast majority of the sample yielded structures that matched the design; however, there are areas where the lift-off procedure was incomplete or became attached to the surface and is one source of discrepancy between the experiment and simulation.

\section{Measurements}

Figures 3 and 4 show the simulated and measured emittance, $\varepsilon(\lambda, \theta, \varphi)$. Emittance can be calculated from simulated and measured reflectivity using Kirchhoff's law:

$$
\varepsilon(\lambda, \theta, \varphi)=\alpha(\lambda, \theta, \varphi)=1-\rho(\lambda, \theta, \varphi),
$$

where $\alpha$ is the absorptance and $\rho$ is the measured reflectance. Because of the ground plane, there is no transmittance through the sample.

Previous experimental studies directly measuring the emitted power have demonstrated this analysis to be valid for similar structured surfaces. ${ }^{13}$ A SOC-100 (surface optics) HDR was used to measure the reflectance. This uses an incoherent source to illuminate a gold-coated hemisphere, which then focuses the radiation toward the sample at a solid angle of $2 \pi$, illuminating the sample isotropically. The reflected light can then be collected at any polar angle between $\sim 10 \mathrm{deg}$ and $80 \mathrm{deg}$. Figures 3 shows the simulated and measured emittance as a function of wavelength, polar angle, and azimuthal angle, $\varepsilon(\lambda, \theta, \varphi)$. Qualitatively there is reasonable agreement between simulation and measurement; although some of the peaks are shifted slightly, the principal features predicted by the model can be observed experimentally. Unfortunately, optical 


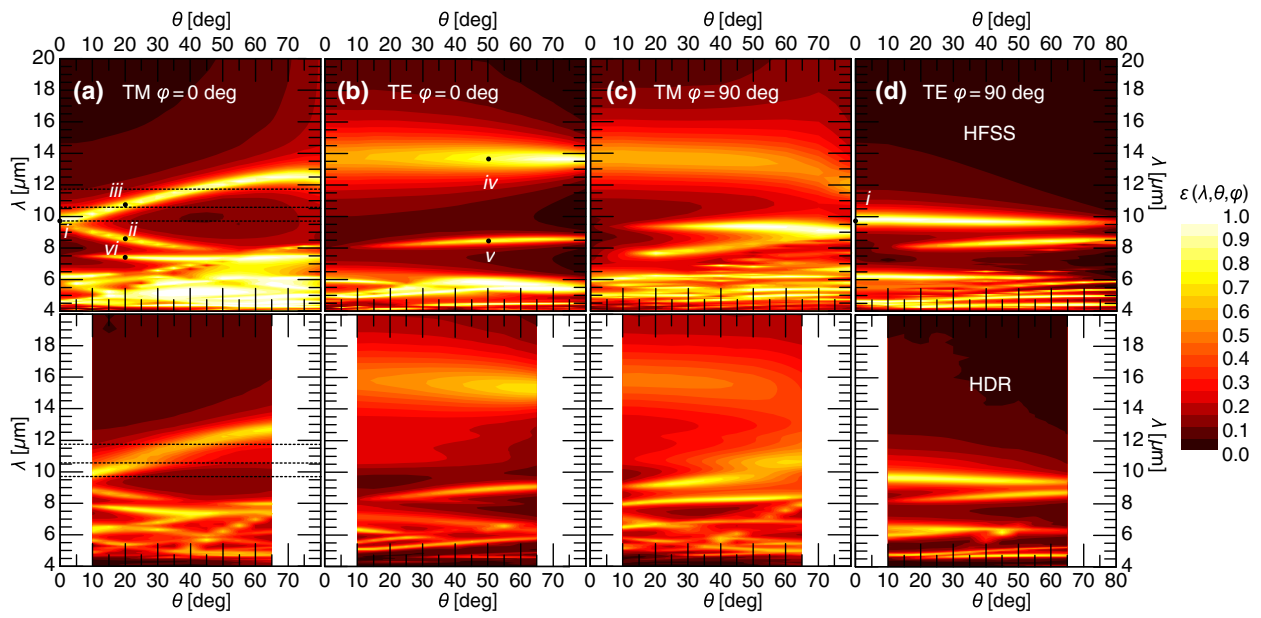

Fig. 3 Comparison between HFSS simulated (top row) and hemispherical directional reflectometer (HDR) experimentally measured (bottom row) spectral/directional emittance: (a) $\varphi=0$ deg transverse magnetic (TM), (b) $\varphi=0$ deg transverse electric (TE), (c) $\varphi=90$ deg TM, and (d) $\varphi=90 \mathrm{deg}$ TE.

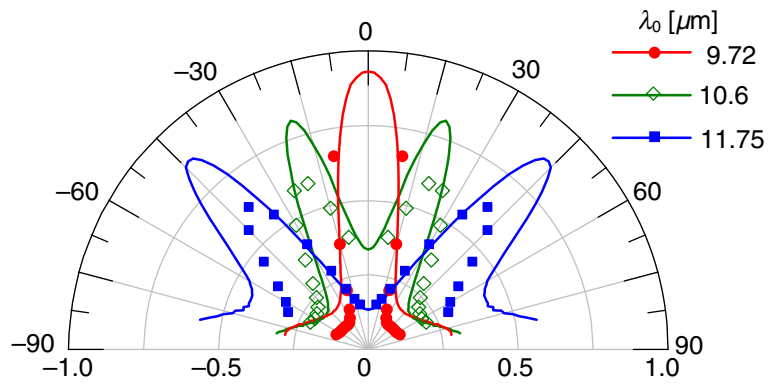

Fig. 4 Angular TM absorptance at three different wavelengths for backward propagating branch, solid lines indicate HFSS simulation and points are measured data.

components used in the measurement apparatus limit the HDR from taking data at normal incidence.

This structure forms a leaky-wave antenna and supports both backward and forward propagating branches.$^{20}$ These branches intersect at broadside $\left(\theta=0 \mathrm{deg}, \lambda_{0}=9.72 \mu \mathrm{m}\right)$. Of note is the Fano-type interference between the forward propagating mode and a patch resonance at $\theta=$ $35 \operatorname{deg} \lambda_{0}=8 \mu \mathrm{m}$ in Fig. 3(a), which is evident in both simulation and experiment.

Figure 4 shows a comparison between measured and simulated angular emittance at discrete wavelengths for TM polarization [dashed lines in Fig. 3(a)]. The beam width at $\lambda_{0}=$ $9.72 \mu \mathrm{m}$ is $\Delta \theta=20$ - deg FWHM. The effective length, $L$, of the leaky-wave antenna can be approximated as ${ }^{20}$

$$
L \approx \lambda_{0} /\left(\Delta \theta \cdot \cos \theta_{m}\right),
$$

where $\theta_{m}$ is the angle of the maximum of the beam measured from normal incidence and $\Delta \theta$ is the beam width in radians. At $\lambda_{0}=9.72 \mu \mathrm{m}$, this corresponds to $L=27.8 \mu \mathrm{m}$ or $\sim 7$ unit cells of the FSS. The figure shows that the array couples light off-axis for the backward propagating leaky-wave branch when the wavelength is greater than the broadside wavelength.

The experiment validates the simulation sufficiently that we can use the numerical model to understand the modal structure that produces the directional emittance. The leaky-wave mode is evident in Fig. 3(a). This mode is forward propagating for shorter wavelengths (ii) and backward propagating for longer wavelengths (iii). Figure 5 shows the simulated field distributions on the $x z$-plane for points on these branches and where they converge to form a single beam [point $i$ in Figs. 3(a) and 3(d)]. This mode is also excited under transverse electric (TE) illumination with 
(a)

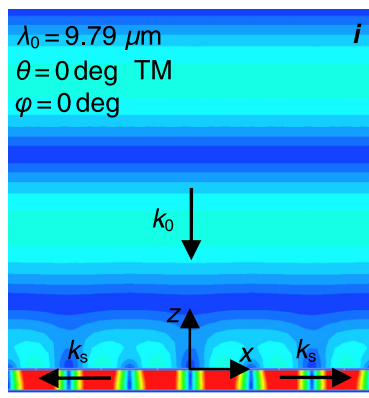

(b)

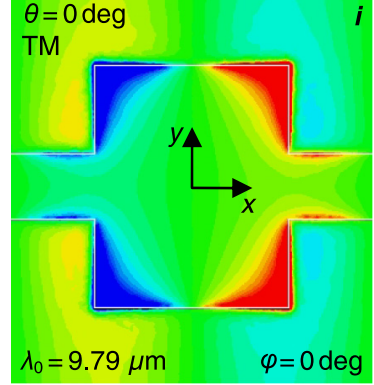

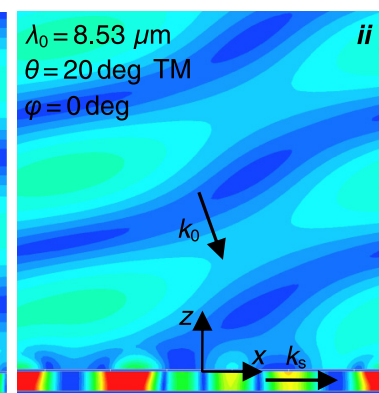

$\theta=50 \mathrm{deg}$

TE

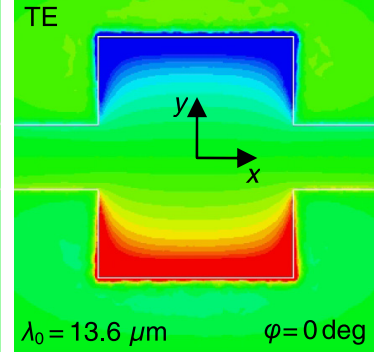

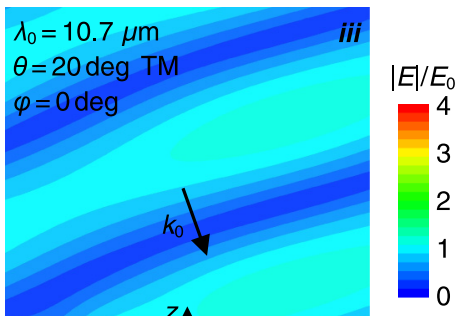

$|E| / E_{0}$
4
3
2
1
0
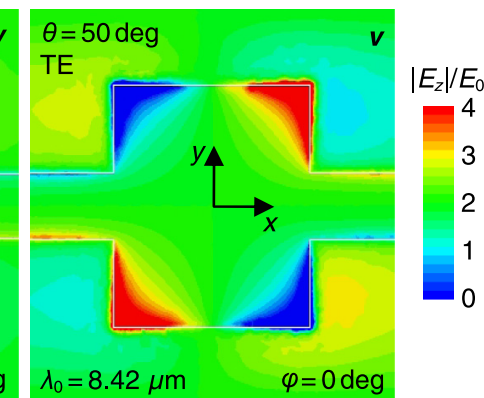

Fig. 5 Selected near-field modes corresponding to points in Fig. 3: (a) normalized magnitude of the E-field for points $i$, ii, and iii, and (b) normalized magnitude of z-component of E-field $5 \mathrm{~nm}$ above FSS for points $i, i v$, and $v$.

$\varphi=90 \mathrm{deg}$. The broad angular-independent mode in the TE plots $\sim 14 \mu \mathrm{m}$ corresponds to the patch mode of the structure polarized in the $y$-direction $(i v)$. Because the patches are not connected with microstrips in this direction, there is significantly lower coupling and no coherence, so the FSS does not create an angular dependence. Figure 5 also shows the second-patch quadrupole mode which is sensitive to wavelength but not polarization [the $v$ branch is visible for the TE illumination conditions in Figs. 3(b) and 3(d)]. Because of symmetry, the quadrupole mode is not excited at normal incidence.

To illustrate how a thermally generated electric current can couple to the structure, we also modeled the simulation with a local excitation. Figure 6 shows the system when it is driven by a local current source. The current source is 500-nm long and centered on a patch. It is oscillating
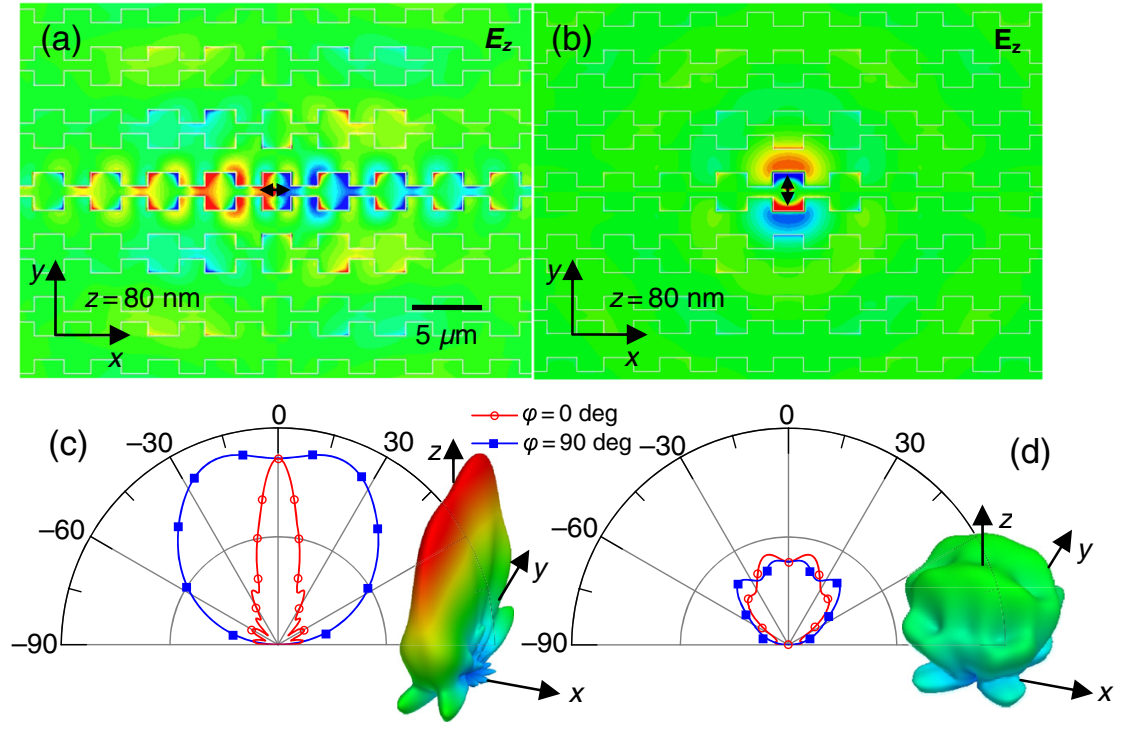

Fig. 6 Long-wave (LW)-FSS excited by a local current source. $E_{z}$ for current excitation in the (a) $x$-direction (b) $y$-direction. Far-field pattern for current excitation in the (c) $x$-direction and (d) $y$-direction. 
at $30.84 \mathrm{THz}(\lambda=9.72 \mu \mathrm{m})$. Figures $6(\mathrm{a})$ and $6(\mathrm{~b})$ show the electric field in the $z$-direction, $5 \mathrm{~nm}$ above the patches, when the current is in the $x$ - and $y$-directions, respectively. Figures 6(c) and 6(d) show the far-field patterns for the antenna system, respectively. When the current is polarized in the $x$-direction, the energy is coupled along the microstrips to multiple patch antennas in agreement with the results from Eq. (3). This produces a much larger effective aperture for the antenna than a single patch, producing a fan-shaped beam in agreement with Fig. 3. When the current is polarized in the $y$-direction, there is minimal coupling of the adjacent patches which produces a more isotropic far-field pattern.

\section{Conclusion}

This paper demonstrated directional thermal emission emanating from enhanced coherence provided by waveguide modes. The ability to control the spectral and directional emittance profiles of surfaces has significant applications for radiation heat transfer and sensing. The patch antennas that form the basis of this work are relatively broadband. Further selectivity can be achieved by using narrowband antennas. This work was conducted at room temperature but can be extended to systems that can efficiently radiate energy at temperatures more applicable to heat-transfer applications.

\section{Acknowledgments}

The authors acknowledge Dr. Eric Tucker for measuring the emittance of the FSS with the SOC-100 HDR.

\section{References}

1. M. F. Modest, Radiative Heat Transfer, Academic Press, San Diego (2003).

2. J.-J. Greffet et al., "Coherent emission of light by thermal sources," Nature 416, 61-64 (2002).

3. M. Laroche et al., "Highly directional radiation generated by a tungsten thermal source," Opt. Lett. 30(19), 2623-2625 (2005).

4. A. A. Cruz-Cabrera et al., "Demonstration of thermal emission control," Proc. SPIE 7591, 75910P (2010).

5. B. A. Munk, Frequency Selective Surfaces, Theory and Design, Wiley-Interscience, New York (2000).

6. J. A. Mason, S. Smith, and D. Wasserman, "Strong absorption and selective thermal emission from a midinfrared metamaterial," Appl. Phys. Lett. 98, 241105 (2011).

7. J. A. Bossard et al., "The design and fabrication of planar multiband metallodielectric frequency selective surfaces for infrared applications," IEEE Trans. Antennas Propag. 54(4), 1265-1275 (2006).

8. D. J. Shelton et al., "Gangbuster frequency selective surface metamaterials in terahertz band," Electron. Lett. 44(22), 1288-1289 (2008).

9. J. Ginn et al., "Planar infrared binary phase reflectarray," Opt. Lett. 33(8), 779-781 (2008).

10. N. Yu et al., "Light propagation with phase discontinuities: generalized laws for reflection and refraction," Science 334, 333-337 (2011).

11. S. Wadsworth and G. D. Boreman, "Broadband infrared meanderline reflective quarterwave plate," Opt. Express 19(11), 10604-10613 (2011).

12. J. Ginn et al., "Polarized infrared emission using frequency selective surfaces," Opt. Express 18(5), 4557-4563 (2010).

13. B. Monacelli et al., "Infrared frequency selective surface based on circuit-analog square loop design," IEEE Trans. Antennas Propag. 53(2), 745-752 (2005).

14. X. Liu et al., "Taming the blackbody with infrared metamaterials as selective thermal emitters," Phys. Rev. Lett. 107, 045901 (2011).

15. I. Codreanu et al., "Microstrip antenna-coupled infrared detector," Electron. Lett. 35(25), 2166-2167 (1999). 
16. C. Middlebrook et al., "Infrared phased-array antenna," Microwave Opt. Technol. Lett. 50(3), 719-723 (2008).

17. T. Kosako, Y. Kadoya, and H.F. Hofmann, "Directional control of light by a nano-optical Yagi-Uda antenna," Nat. Photonics 4(5), 312-315 (2010).

18. B. A. Slovick et al., "Directional control of infrared antenna-coupled tunnel diodes," Opt. Express 18(20), 20960-20967 (2010).

19. J. Ginn et al., "Modeling infrared frequency selective surfaces with frequency dependent materials," in 22nd Int. Review of Progress in Applied Computational Electromagnetics (ACES), pp. 307-311 (2006).

20. J. L. Volakis, Ed., Antenna Engineering Handbook, 4th ed., McGraw-Hill, New York (2007).

Edward C. Kinzel received his BS, MS, and $\mathrm{PhD}$ degrees from Purdue University in mechanical engineering. From 2011 to 2012, he was a postdoc in Dr. Glenn Boreman's lab at University of Central Florida (CREOL) and at UNCC. In 2012, he joined the faculty of the Mechanical and Aerospace Engineering Department at the Missouri University of Science and Technology in Rolla, Missouri. His research focuses on the intersection of optics, heat transfer, and manufacturing.

James C. Ginn graduated from the University of Central Florida with his $\mathrm{PhD}$ in electrical engineering in 2009. At the university, he carried out research in infrared planar focusing surfaces, polarized thermal emission surfaces, low-power rf energy harvesting, and genetic algorithm optimization of microwave antenna devices. He has co-authored over 50 journal papers and proceedings. He currently specializes in numerical analysis of electromagnetic structures, optical ellipsometry, and nano-fabrication as Plasmonics, Inc.'s senior executive vice president and CTO.

Louis A. Florence: Biography is not available.

Brian A. Lail received his BS degree in physics from Furman University, MS degree in physics and electrical engineering, and his $\mathrm{PhD}$ in electrical engineering from New Mexico State University. From 2002 to 2005, he was with the Department of Electrical and Computer Engineering at the University of Central Florida. Since 2005, he has been with the Department of Electrical and Computer Engineering at Florida Institute of Technology, where he is currently an associate professor, engaged in teaching and conducting research in applied and computational electromagnetics.

Glenn D. Boreman is professor and chair of the Department of Physics and Optical Science, University of North Carolina at Charlotte. From 1984 to 2011, he was a member of the faculty of the University of Central Florida, where he supervised $25 \mathrm{PhD}$ students to completion. $\mathrm{He}$ received his BS degree in optics from University of Rochester, and his $\mathrm{PhD}$ in optics from University of Arizona. He served as editor-in-chief of Applied Optics, and is presently deputy editor of Optics Express. He is a fellow of OSA and SPIE, and is the 2016 president-elect of SPIE. 